\title{
Quantifying Natural Fault Geometry: Statistics of Splay Fault Angles
}

\author{
Ryosuke Ando ${ }^{1,}$, , Bruce E. Shaw ${ }^{1}$ and Christopher. H. Scholz ${ }^{1}$ \\ 1 Lamont-Doherty Earth Observatory, Columbia University, Palisades, NY, USA \\ * Now at National Research Institute for Earth Science and Disaster Prevention, Tsukuba, Ibaraki, \\ Japan
}

\begin{abstract}
We propose a new approach to quantifying fault system geometry, using an objective fit of the fault geometry to a test function, specifically here a fault branch. Fitting a "Y" shaped object using a cost function to dextral faults in California, we find a number of significant results arising from using a systematic, objective, quantitative approach. (1) The largest angle of the branch structure is generally very close to $180^{\circ}$, implying the branch is a splay fault off the primary throughgoing fault. (2) The distribution of the smallest angle, the splay angle, has a peak near $\pm 17^{\circ}$, symmetric about the primary fault. (3) These features appear independent of scale. These results are not yet explained by any theory, and pose new questions and constraints for the physics of fault system formation and behavior.
\end{abstract}

\section{Introduction}

Faults do not act as isolated objects, but rather as parts of complex fault systems. Quantifying the geometry of fault systems remains an important and in many ways an unsolved problem. Here, we propose a new approach to the problem, using objective criteria to match geometrical objects to mapped faults. Specifically, we focus on a geometrical object of a branching fault, and numerically fit with a cost function the mapped fault system to this geometrical object. This approach allows for a systematic, unbiased, quantitative measure of a significant aspect of fault system geometry.

We examine the particular geometrical object of fault branches for a few reasons. One reason is it is a geometrical object which does not have an explicit scale, and since fault structures appear similar from ranges of hundreds of meters to hundreds of kilometers, we would like to find scale-independent measures of geometry—and, moreover, test the apparent scale invariance.

Fault branches are not, of course, the only relevant geometrical irregularities-fault stopovers (Wesnousky 2006) and bends (King and Nabelek 1985) being other examples. These geometrical 
irregularities play an important role in earthquake behavior and are used to "segment" faults to try to understand fault and earthquake behavior.

Fault segmentation is seismologically important in controlling earthquake rupture processes including nucleation, propagation and termination of ruptures (King and Nabelek 1985; Shaw 2006). Splay faults are particularly important for dynamic fault segment interactions and earthquake rupture path selectivity. There are examples in recent earthquakes that ruptures branched off into splay faults from primary faults from the evidence of surface rupture traces such as the 1992 Landers earthquake (Sowers et al. 1994), the 2001 Kunlun Earthquake (Xu et al. 2006) and the 2002 Denali Earthquake (Eberhart-Phillips et al. 2003). A difference in a rupture path leads to differences in seismic hazards and, further, splay fault ruptures may cause disastrous damage; e.g. it is thought that the rupture propagation into an offshore upward splay fault branching from a subduction plate boundary was responsible for intensive Tsunami generation during the 1944 Tonankai Earthquake (e.g. Park et al. 2002). Several recent theoretical studies suggest that the one of the key parameters to determine the rupture extension into splay faults is the splay angle (Ando and Yamashita 2007; Bhat et al. 2007; Kame et al. 2003). However, the general characteristics of splay fault geometry are poorly understood. Here we quantify splay angles for the case of strike-slip faults.

The quantification of natural fault geometries using mapped surface traces have been widely conducted, however, these analyses mostly dealt with lengths or separation distances of fault segments (e.g. Cowie et al. 1996) or the roughness (topography) of fault surfaces (e.g. Brown and Scholz 1985).

The difficulties in the analysis of splay faults should be in the definition of objective criterion to geometrically quantify the structure. For instance, regarding the splay angle, the value may change depending on the observer's visual perception: one could lay a protractor along different portions of a rough fault segment. The size of the protractor may also affect the results. Dealing with large number of data is another difficulty since the fault geometry is usually analyzed by visual and manual procedures.

In order to overcome these difficulties, we propose an objective method to quantify splay structures based on a pattern matching analysis using a computer. The pattern matching is a standard methodology in computer-based geometrical pattern recognition and data mining algorithms where we search for a certain geometry that is similar to a test function prepared in advance to mimic a targeted geometry. To model the splay fault geometry, we employ as the test function in this study a set of three line segments connected to each other at one end. The degree of matching can be 
evaluated in terms of an index similar to a correlation coefficient so that the analysis becomes quantitative and objective.

In this paper we concentrate on the presentation of our methodology and our observational results; Modeling of what we observe will be done in subsequent papers. The purpose of this paper is to present geometrical characteristics that are found for the first time.

\section{Method}

We employed a pattern matching algorithm for the automatic detection of splay fault structures. In our method, any part of fault traces in the data is compared with a test function prepared in advance, and the degree of their similarity is evaluated, and finally test functions well fitted to the fault traces are retained. For the geometry of the test function, we simply implement a set of three line segments with the same length that are connected with each other at a point. To adopt any splay angles in the fault traces, we allow any orientation for the assumed line segments. Examples of test functions fitted to fault traces are shown in Figs. 1 and 2.

The shape of the test function is defined by the length of each line segment $R$ and the two angles $\Phi_{p}$ and $\Phi_{s}$, which respectively denote the angles made by the most separated and the closest two line segments (The subscripts $\mathrm{p}$ and $\mathrm{s}$ are chosen as they will be found to be associated with the primary and splay faults, respectively). For instance, if the test function is shaped like "T", $\Phi_{p}=180^{\circ}$ and $\Phi_{s}=90^{\circ}$. As shown later, we can measure not only the splay angles through $\Phi_{p}$ and $\Phi_{s}$ but also the scale length of the splay faults through $R$.

The degree of similarity between the test function and the fault traces is quantified in terms of the index $F$ defined as follows. For simplicity, we define $F$ for an individual line segment not for the whole test function consisting of the three line segments. In this sense, $F$ is defined as

$$
F=\frac{1}{R} \int_{0}^{R} \exp \left\{-\left(\frac{\min \left(\mathbf{r}-\mathbf{r}^{\prime}\right)}{R}\right)^{2} / \sigma_{r}-\left(\frac{\theta-\theta^{\prime}}{\pi}\right)^{2} / \sigma_{\theta}\right\} d r,
$$

where $\mathbf{r}(r)$ and $\mathbf{r}$ ' are respectively the location on this line segment and a point on the fault trace; the function $\min \left(\mathbf{r}-\mathbf{r}^{\prime}\right)$ gives $\mathbf{r}^{\prime}$ that is the closest to $\mathbf{r} . \theta$ and $\theta$ ' represent the orientations of the normal vectors for this line segment and the fault trace at locations $\mathbf{r}$ and $\mathbf{r}$ ' respectively. The integration is taken over $r$, the distance along this line segment. Since the integrand is the shape of a Gaussian function, if the line segment completely overlaps with a fault trace, $F$ takes 1 , contrary if both are far apart, $F$ becomes 0 . The width of the Gaussian is controlled by $\sigma_{r}$ and $\sigma_{\theta}$ so that the sharpness of the 
fit is also controlled, where smaller or larger values give tighter or looser fit. To allow loose fit by some extent with $\sigma_{r}$ and $\sigma_{\theta}$ is, in fact, essential in this study since the actual fault geometry is not a straight line but still can be reasonably approximated by the line. In other words, the fault roughness is absolved by a lobe existing around the line segment of the test function, which corresponds with the skirt of the Gaussian.

For the sake of implementation, we need to expand the above matching procedure supposing single line segment to a procedure assuming the test function with three arms. We follow two stages in the procedure. As the initial stage, first, we chose an arbitrary point on the fault in the data to locate the center of the test function. Next, we again pick up one line segment whose one end is fixed at this chosen point, and evaluate its matching with the fault geometry at any orientation of it. In this way, we can obtain the index $F(\varphi)$ as the function the orientation $\varphi\left(0<\varphi<360^{\circ}\right)$. The orientations corresponding three largest peaks in the $F(\varphi)$ distribution become the possible orientation of the three arms of the test function. Then, the threshold $F_{c}$ is applied to the three largest peaks for their values $P_{1}, P_{2}$ and $P_{3}$. If all of these peak values exceed $F_{c}$, this test function is qualified in the initial stage. In the second stage, first, we iterate the initial stage to provide the candidates of the qualified test functions along the fault traces. Second, we select the most qualified candidates within certain neighborhoods, which are defined as the test functions having the largest values of $P_{1}+P_{2}+P_{3}$ with them; specifically, to determine the size of the neighborhood, we assume the minimum distance between the qualifiers $R_{o}$. Finally, we can obtain a set of test functions well fitted to the fault trace geometry in the data.

In addition, we would like to mention that this method is readily applicable to raster formatted data as well as vector formatted data set used in this study. Rasterised data is more easily obtained by digital images. Thus it will be interesting to apply this method to such data including digitized hand-drawn sketches and pictures taken in the field or laboratory.

\section{Results}

We analyzed the splay angle of faults in California, USA, using the digital database of quaternary active faults (Bryant 2005). This database includes the location of 18730 fault segments represented in vector format data points with spatial resolution of a few hundred meters. Although the data is only about the surface trace, it can be used to represent the geometry of strike slip faults at depth through the seismogenic layer since their dip angles tend to be 90 degrees. We choose to use the catalogued dextral faults, which are dominant due to the tectonic setting in this region. To avoid near surface characteristics in the fault geometry, we focus on fault structures larger than $5 \mathrm{~km}$ by 
choosing $R$ values which are a substantial fraction or larger of the seismogenic layer (Scholz 2002) and can be considered as major persistent structures throughout this layer.

We begin with the case of $R=20 \mathrm{~km}$, which is the length of each line segment of the test function. The surface traces of these dextral faults in the catalog are plotted in Fig. 1 with fitted fault geometries or test functions obtained by the present pattern matching analysis. We select a set of parameters $F_{c},=0.4, \sigma_{r}=0.001$ and $\sigma_{\theta}=0.05$. These values of the parameters are confirmed to return the most appropriated fitting results after a systematic parameter study, which we will discuss further later.

First of all, we demonstrate the reliability of the method and analysis results. The quality of the fitting can be confirmed in the zoom shown in Figs. $1 \mathrm{~b}$ and 1c for central and southern California respectively. We see that each line segment of the fitted fault geometry follows corresponding fault traces in the catalog, and splay points in the fault traces are centered by the junctures of the fitted test functions. As we stated in the previous section, it is also confirmed that the fitted line segments are located on the center of oscillating fault traces, with the amplitudes of the oscillation being reasonably small as seen in the figures. The performance in detecting splay fault locations is also satisfactory as well, which can track nearly every splay faults supposed to be marked. Note that the fitted fault geometries are exclusive within the area $R_{o}=0.25 R$ (throughout the analysis); some splays in densely distributed areas are therefore not picked due to this restriction.

Before detailed investigations, it is worth characterizing the overall splay fault locations and geometry. Fig. 1a shows that most of the fitted fault geometries are closely placed near the San Andreas Fault system (SAF). Moreover, the splay faults are particularly localized in the central and southern California regions along the SAF as boxed in Fig. 1a. Contrarily, the rest of the areas have much less splay faults and the dextral fault geometry appears to be much simpler in these areas.

In central California (Fig. 1b), the fitted fault geometries are closely located near the junctions of SAF and its major splays, the Calaveras Fault (CF) and San Gregorio Fault (SGF). There are interesting characteristics observed in this area; these major splays are oblique to the SAF and parallel with each other, but smaller splays are not. These splays do not seem to cut the primary faults. In southern California, by contrast to northern CA, a cluster of minor fault segments exists apart from the SAF, which is called the Mohave Shear Zone (MSZ). The distributions of the fitted fault geometries are shown to be denser in the MSZ than region along the SAF and its major splays, the San Jacinto Fault (SJF) or the Elsinore fault (EF). 


\section{Scale dependence and independence of splays}

The analysis is further applied to different scales over a decade from $5 \mathrm{~km}$ to $50 \mathrm{~km}$ assuming various lengths of the test function $R$. Examples in southern California are shown in Fig. 2. First, we should emphasize that the fault structures of specific length scales can be detected by the proper choice of $R$. For instance, at the junction of SAF and SJF located near $(X, Y)=(1.5,1)$, this position is not recognized as the location of a splay for the cases of $R=5 \mathrm{~km}$ (a) and $15 \mathrm{~km}$ (b) due to the existence of a gap of about $4 \mathrm{~km}$ width between them, while this gap is small enough for the cases of $R=30 \mathrm{~km}$ (c) and $50 \mathrm{~km}(\mathrm{~d})$ where the splay faults are marked. Contrary, smaller structures are captured by smaller $R$ cases. On the contrary, the four small splay faults, located along SAF within $X=0.5$ to $1.0 \times 10^{\wedge} 5 \mathrm{~m}$ and $Y=1$ to $1.5 \times 10^{\wedge} 5 \mathrm{~m}$, are large enough for the case of $5 \mathrm{~km}$ but too small for the cases of $\mathrm{R}>5 \mathrm{~km}$. These observations demonstrate that this method can capture the fault structures of desired scales with the adjustment of $R$.

The scale dependence observed in the number of splay faults $N_{R}$ is a significant characteristic as roughly recognized in Fig. 2a-d. By counting the number for the entire California, we found that $N_{R}$ is a decreasing function of $R$ as $N_{R}=137,97,67,50,39,34$ and 27 respectively for $R=5,10,15,20$, 30,40 and $50 \mathrm{~km}$. It is interesting that $N_{R}$ appears to follow a power law function over a decade in scale length, but this is too limited a scale range to really determine such functional forms. We can also observe in Fig. 2 that splays themselves can have smaller splays. This appears to coincide with the well known fractal geometry of natural faults (Aviles et al. 1987; Okubo and Aki 1987).

\section{Statistics of primary and splay fault angles}

For angles made by three arms of each fitted test function in Figs. 1 and 2, we can recognize a quite common characteristic on the geometry of splay fault structure regardless of the scale and the region. That is two of three arms fall into almost a straight line. This is supported by a statistical analysis as shown in Fig. 3; we can see that $\Phi_{p}$ (see the inset and section 2 for the definition) has a well defined peak at about $180^{\circ}$ in the statistical distribution over the entire California. This implies that "Y"-like shapes of symmetric branches is very rare in natural fault geometry. So far, we use the term of splay faults without the definition. But our analysis clearly demonstrates that the majority of bifurcated structures in natural faults are actually what has been inferred from splay faults by experiences in the field, which consist of planar primary faults and subsidiary oblique splay faults. This geometric asymmetry is an important clue to model the formation of the splay faults.

The statistical analysis is finally applied to evaluate the obtained angle of the splay faults for the 
entire California. The splay fault angle technically corresponds to the angle between the two closest line segments of the test function, $\Phi_{s}$. The probability distributions of the obtained splay angles $\Phi_{s}$ are shown in Fig. 4 for various $R$. The sign of the angle distinguishes the orientation of a splay; $\Phi_{s}$ is measured from the primary fault clockwise so that plus/minus denotes splays located on the right/left hand side of a primary fault (see the inset).

In Fig. 4, there are four significant properties in the distribution of the splay angles $\Phi_{s}$.

1. The splay angle distributions show sharp peaks at about $-/+17^{\circ}$ (refer the caption for the median of each case).

2. Unexpectedly, the distributions of the left and right sides are remarkably symmetric, with the splays symmetrically distributed on either side of the primary faults.

3. The splay angle distributions for any $R$ cases are similar in their shapes and peak values. This means that the geometrical relations between a primary fault and a splay fault are self-similar, independent of the length of the splay faults.

4. The individual splay angle distribution is asymmetric, with the peak angle close to its minimum value. In other words, the minima are sharply defined but the maxima are not.

Some roughness seen in the distribution of large $R$ cases in Figs. 3 and 4 should be mainly due to the relatively small sampling number. It is obviously confirmed that the distributions tend to be much smoother as the sampling number increase. On the other hand, it should be noted that the analysis covers the entire California, which includes various tectonic settings from the SAF as a mature plate boundary to the Mohave shear zone as a cluster of immature faults. Further investigation with division regarding local tectonic conditions may give us more detailed statistics behind the current over-all features. However, the above features in the distribution of the splay angles are still considered to be quite robust and tell us about the fundamental characteristics of splay fault structures.

The first and second features in the above imply that the fault tip stress field may not play a predominant role on the formation of the splay faults, since its normal stress component is anti-symmetric against the fault plane. Regarding the third feature, it should be mentioned that this can add different perspective and data to the long-standing discussion whether the fault geometry is self-similar or self-affine, and fractal or not; there is a limitation in those discussions as they have 
been mostly based on the observation of fault surface topography (e.g. Brown and Scholz 1985; Sagy et al. 2007) or length of fault segments (e.g. Cowie et al. 1996). Deeper understandings should be necessary for the individual physics of the initiation and evolution of those characteristic structures. In terms of dynamic rupture path selectivity, referring Kame et al. [2003], the observed splay angle $17^{\circ}$ suggests that a rupture tends to propagate to either primary or splay faults. But if the rupture speed is almost at the terminal (Rayleigh wave speed), the rupture can be bifurcated to both.

\section{Validation of the results}

We validate the robustness of the obtained results in terms of the parameters for the threshold $F_{c}$ and the sharpness of the fit $\sigma_{r}$ and $\sigma_{\theta}$. Fig. 3b-d shows these effects on the obtained splay angles, where the sign of the angles are not considered.

We first find from Fig. 5a that the choice of $F_{c}$ does not affect strongly the obtained splay angles as far as we apply proper $F_{c}$ that gives statistically meaningful number of splays. For instance, the cases of $F_{c}<0.7$ shows quite similar result with sampling 55 splays in the case of $F_{c}=0.4$ and $R=20 \mathrm{~km}$, contrary, $F_{c}=0.7$ showing different is a case of poor statistics picking only 8 splays. The choice of threshold value sometimes can be a problem in pattern matching, however, this analysis shows quite stable behavior over a wide range of the parameter value.

For the sharpness parameters, we find that as seen in Fig. $5 \mathrm{~b}$ variation of $\sigma_{r}$ within half and double of the most optimal value could lead the relatively large differences up to $10^{\circ}$. However, fortunately, the variation in the quality of the fitting is also significantly noticeable when checking the fits on map by eyes: too large value (loose fit) recognize parallel strands as splays by mistake, which also causes higher splay angles to appear in the statistics, while too small value (tight fit) tends to miss obvious splays due to their roughness in the geometry. In contrast, variation in $\sigma_{\theta}$ (Fig. 5c) has a smaller effect although its implementation is of great help to reduce mistakes in densely distributed areas. Thus the parameter tuning to obtain the most optimal fit is quite straightforward and the obtained results become reliable once $\sigma_{r}$ is chosen properly. It should be emphasized that the obtained results are quite robust because of the stableness enabled by this method.

\section{Acknowledgements}

R. A. is supported by Japan Society for Promotion of Science Postdoctoral fellowship in 2005-2007. B.E.S. is supported by NSF grant EAR03-37226 and by the Southern California Earthquake Center. 


\section{Captions}

Figure 1. Surface faults and fitted fault geometry. Plots for (a) the entire California and the zooms of the boxed areas for (b) northern and (c) southern California. White and black lines represent catalogued faults and fitted fault geometry (test function) respectively. $R=20 \mathrm{~km}$.

Figure 2. Surface faults and fitted fault geometry. Plots for different scales (a) $R=5$, (b) 15, (c) 30 and (d) $50 \mathrm{~km}$ in southern California.

Figure 3. Probability distribution of primary fault bend angles for different scales. Plot was made at intervals of $3^{\circ}$. The medians of the cases of $R=5,15,30$ and $50 \mathrm{~km}$ are 178, 179, 180 and 181 degrees.

Figure 4. Probability distribution of splay fault angles for different scales. The medians of the cases of $R=5,15,30$ and $50 \mathrm{~km}$ are $-17 / 16,-17 / 18,-12 / 16$ and $-17 / 17$ degrees for left/right-hand side branches defined in the inset.

Figure 5. Medians of splay angles for different (a) $F_{c}$, (b) $\sigma_{r}$ and (c) $\sigma_{\theta}$.

References

Ando, R., and T. Yamashita (2007). Effects of mesoscopic-scale fault structure on dynamic earthquake ruptures: Dynamic formation of geometrical complexity of earthquake faults. J. Geophys. Res., 112, doi:10.1029/2006JB004612.

Aviles, C. A., C. H. Scholz, and J. Boatwright (1987). Fractal Analysis Applied to Characteristic Segments of the San-Andreas Fault. J. Geophys. Res., 92(B1), 331-344.

Bhat, H. S., M. Olives, R. Dmowska, and J. R. Rice (2007). Role of fault branches in earthquake rupture dynamics. J. Geophys. Res., 112(B11), doi:10.1029/2007JB005027.

Brown, S. R., and C. H. Scholz (1985). Broad Bandwidth Study of the Topography of Natural Rock Surfaces. J. Geophys. Res., 90(B14), 2575-2582.

Bryant, W. A., compiler (2005). Digital Database of Quaternary and Younger Faults from the Fault Activity Map of California, version 2.0: California Geological Survey Web Page. 
$<\underline{\text { http://www.consrv.ca.gov/CGS/information/publications/QuaternaryFaults_ver2.htm>; }}$ 9.1.2006.

Cowie, P. A., R. J. Knipe, and I. G. Main (1996). Special issue: Scaling laws for fault and fracture populations - Analyses and applications - Introduction. J. Struct. Geol., 18(2-3), R5-R11.

Eberhart-Phillips, D., P. J. Haeussler, J. T. Freymueller, A. D. Frankel, C. M. Rubin, P. Craw, N. A. Ratchkovski, G. Anderson, G. A. Carver, A. J. Crone, T. E. Dawson, H. Fletcher, R. Hansen, E. L. Harp, R. A. Harris, D. P. Hill, S. Hreinsdottir, R. W. Jibson, L. M. Jones, R. Kayen, D. K. Keefer, C. F. Larsen, S. C. Moran, S. F. Personius, G. Plafker, B. Sherrod, K. Sieh, N. Sitar, and W. K. Wallace (2003). The 2002 Denali fault earthquake, Alaska: A large magnitude, slip-partitioned event. Science, 300(5622), 1113-1118.

Kame, N., J. R. Rice, and R. Dmowska (2003). Effects of prestress state and rupture velocity on dynamic fault branching. J. Geophys. Res., 108(B5), doi:10.1029/2002JB002189.

King, G., and J. Nabelek (1985). Role of Fault Bends in the Initiation and Termination of Earthquake Rupture. Science, 228(4702), 984-987.

Okubo, P. G., and K. Aki (1987). Fractal Geometry in the San-Andreas Fault System. J. Geophys. Res., 92(B1), 345-355.

Park, J. O., T. Tsuru, S. Kodaira, P. R. Cummins, and Y. Kaneda (2002). Splay fault branching along the Nankai subduction zone. Science, 297(5584), 1157-1160.

Sagy, A., E. E. Brodsky, and G. J. Axen (2007). Evolution of fault-surface roughness with slip. Geology, 35(3), 283-286.

Scholz, C. H. (2002). The Mechanics of Earthquakes and Faulting, Cambridge Univ. Press, Cambridge, United Kingdom.

Shaw, B. E. (2006). Initiation propagation and termination of elastodynamic ruptures associated with segmentation of faults and shaking hazard. J. Geophys. Res., 111(B8), doi:10.1029/2005JB004093.

Sowers, J. M., J. R. Unruh, W. R. Lettis, and T. D. Rubin (1994). Relationship of the Kickapoo Fault to the Johnson Valley and Homestead Valley Faults, San-Bernardino County, California. Bull. Seismol. Soc. Amer., 84(3), 528-\&.

Wesnousky, S. G. (2006). Predicting the endpoints of earthquake ruptures. Nature, 444(7117), 358-360.

Xu, X. W., G. H. Yu, Y. Klinger, P. Tapponnier, and J. Van der Woerd (2006). Reevaluation of surface rupture parameters and faulting segmentation of the 2001 Kunlunshan earthquake $(\mathrm{M}(\mathrm{w}) 7.8)$, northern Tibetan Plateau, China. J. Geophys. Res., 111(B5), doi:10.1029/2004JB003488. 
Figures
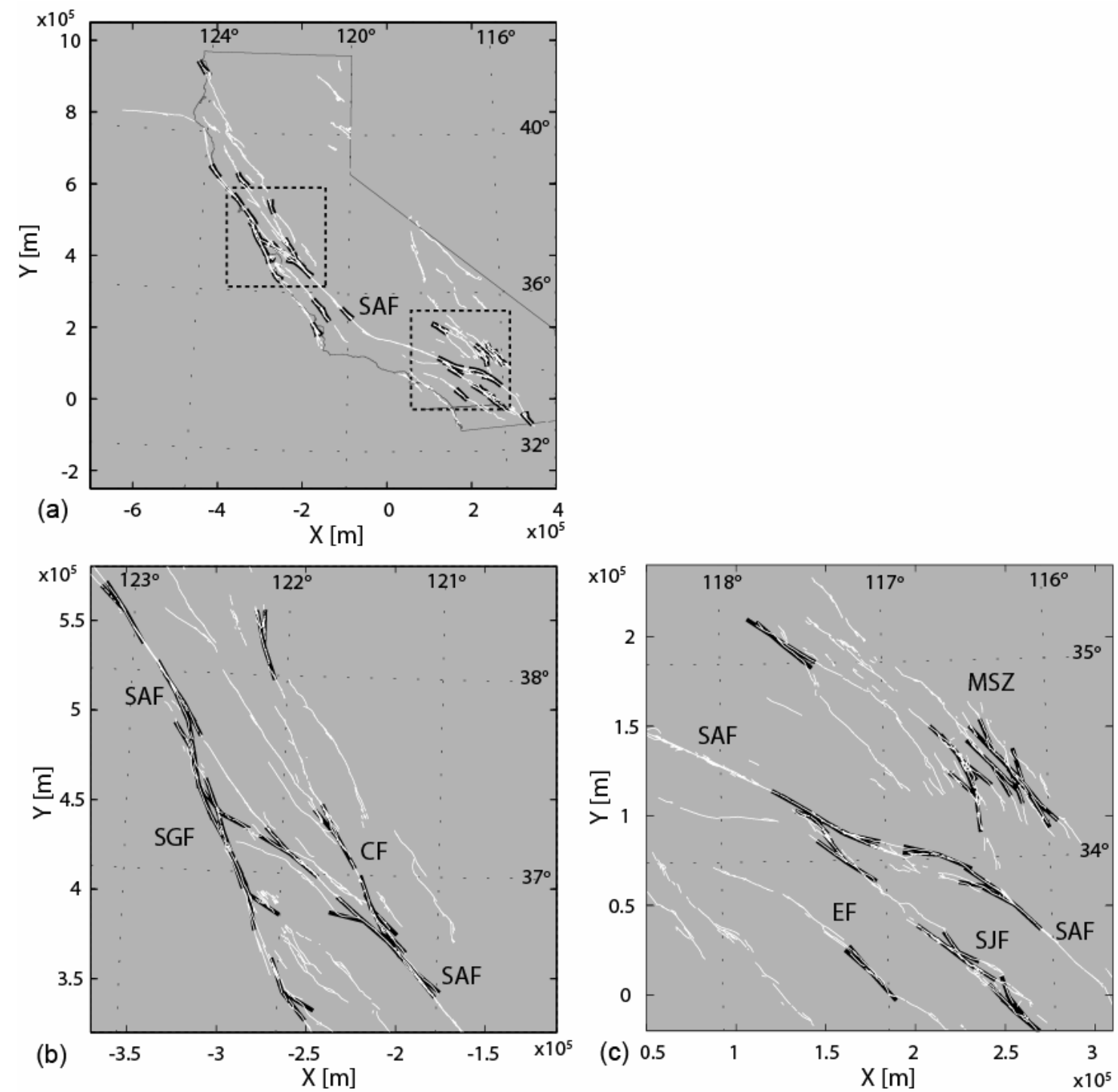

Figure 1. 

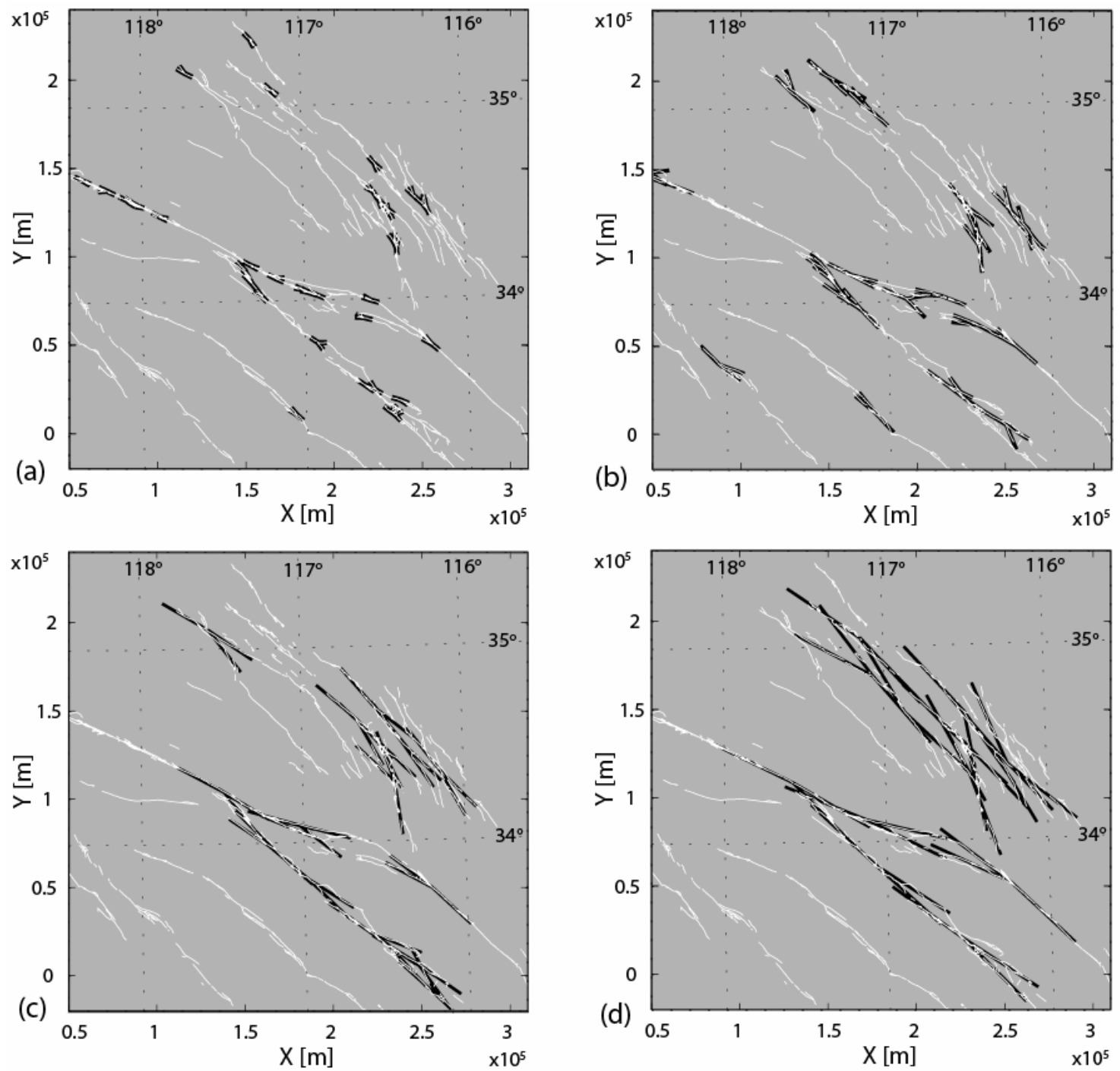

Figure 2 


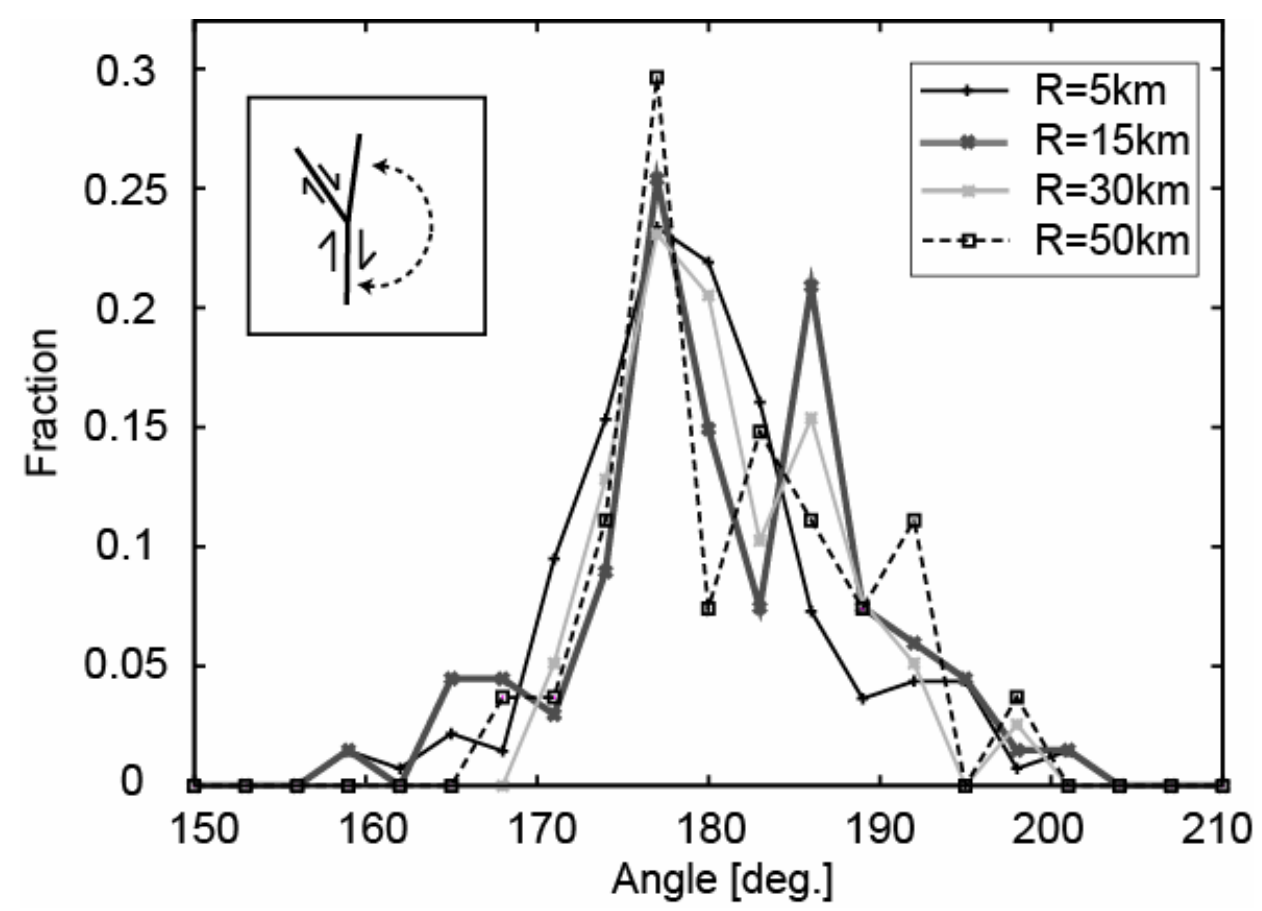

Figure 3.

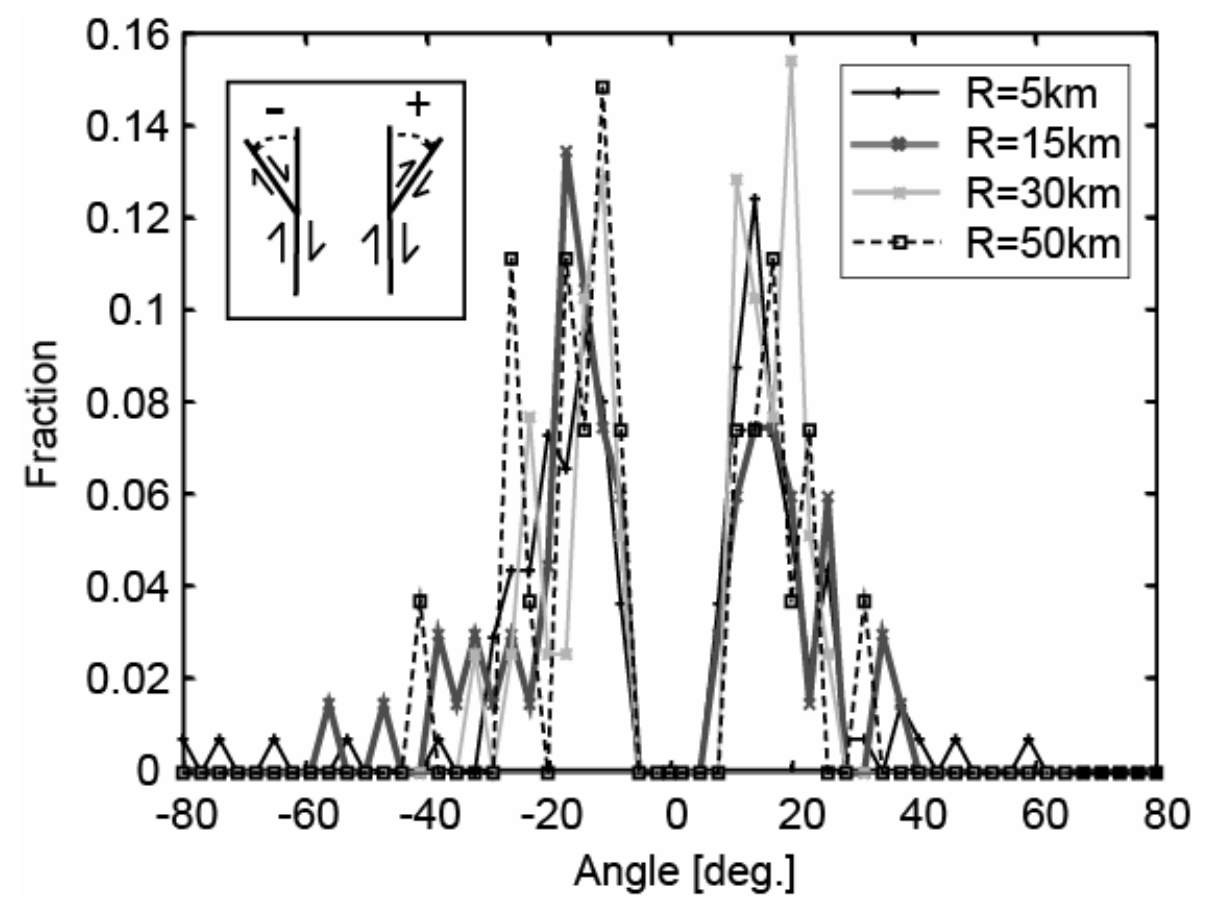

Figure 4 . 


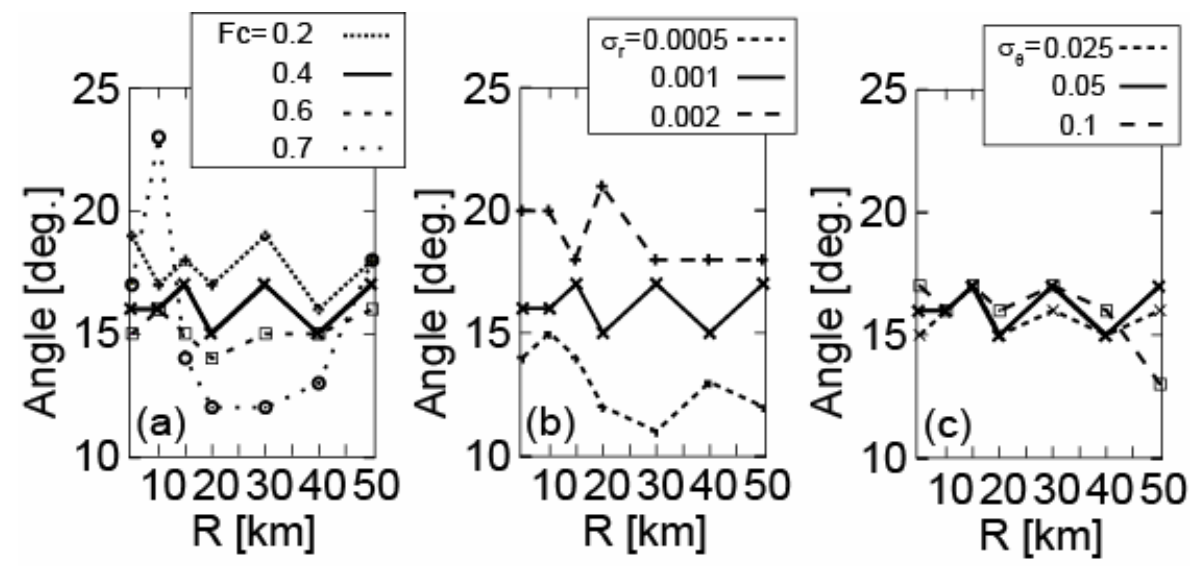

Figure 5. 